\title{
Functions of the corticospinal and corticobulbar tracts in the human newborn
}

\author{
Harvey B. Sarnat \\ Departments of Pediatrics and Pathology, David Geffen School of Medicine at UCLA \\ and Cedars-Sinai Medical Center, Los Angeles, California, U.S.A.
}

\begin{abstract}
The corticospinal and corticobulbar tracts (CST, CBT) are immature at birth, in neuroanatomical terms of myelination and terminal axonal sprouting for multiple synaptic contact; these developmental features are not mature until 2 years of age. Physiologically, the CST is mainly inhibitory. Nevertheless, these pathways have an important role to play in normal neurological function at this age, though different from their functions in the older child and adult. They are important in the neonate by 1) inhibition of the monosynaptic stretch reflexes at spinal cord levels, beginning at 25 weeks gestation or earlier; 2) influence upon muscle tone, hence posture, by mediating proximal flexion in axial and limb girdle muscles and distal extension of the fingers and toes and abduction of the thumbs; 3) antagonism of the proximal extension and distal flexion and adduction from the medial subcorticospinal pathways of Lawrence and Kuypers; 4) reinforcement of tactile reflexes, the most important of which are suck and swallow; 5)early individualization of finger movements; 6) transmission of epileptic activity from the cerebral cortex. Understanding the unique roles of the CST at birth provide rational, physiological explanations of such neonatal phenomena as clonus, opisthotonus, strong distal flexion and
\end{abstract}

Correspondence: Harvey B. Sarnat, M.D., F.R.C.P.C., Cedars-Sinai Medical Center, Departments of Pediatrics (Neurology) and Pathology (Neuropathology), 4221 North Tower, 8700 Beverly Blvd. Los Angeles, CA 90048, U.S.A.

Tel: (310) 423-1299, fax: (310) 423-4131.

Email: harveyb.sarnat@cshs.org

Received: May 16, 2003.

Accepted: June 6, 2003. adduction ("cortical thumb") and fisting), and poor suck and swallow in affected neonates, regardless of the cause of cerebral cortical impairment or its reversibility or irreversibility. In summary, the CST and CBT are indeed important functional pathways in the neonate, but many of their functions are very different than in the adult. (J Pediatr Neurol 2003; 1(1): 3-8).

Key words: corticospinal tract, muscle tone, neonate, posture, stretch reflexes, suck, swallow, tactile reflexes.

\section{Introduction}

It has long been debated in neonatal neurology whether the corticospinal tract (CST) plays a role in the neurological function of the premature and term neonate, or whether the newborn essentially functions reflexively at a brainstem level. The characteristic signs of CST lesions in older children, such as spastic diplegia, rarely are expressed in neonates and usually do not become evident for several months postnatally. Numerous bulbospinal pathways originate in the brainstem and descend to the spinal cord: vestibulospinal, reticulospinal, olivospinal, tectospinal, rubrospinal tracts. These small pathways often are divided into medial and lateral components in the tegmentum of the pons and medulla oblongata, and are believed to be more important in neurological function in the fetus and neonate than at any other time in life, supplanted by the stronger influence of the CST. The question thus is posed as to whether the CST indeed plays any role in the function of the neonatal nervous system, or is essentially silent at this age and gradually begins to mediate cerebral control during the first year. These questions were previously considered by me more than a decade ago (1), and my subsequent experience, both as a clinical neonatal neurologist and as a pediatric neuropathologist, has served to reinforce my earlier conclusions and to supplement 
them with additional observations by me and by other investigators.

The CST is the longest axonal pathway within the central nervous system. Its axonal growth cones have reached their most distant destination, the lowest sacral spinal cord, by midgestation. The maturation of this pathway is not complete until two years of age, however, as defined by two important criteria: 1) myelination; and 2) sprouting of terminal axonal collaterals to make multiple synaptic contacts on both motor neurons and interneurons of the spinal ventral horns. These maturational events begin after 30 weeks gestation. The corticobulbar tract (CBT) is similar in nature, but terminates in brainstem centers. Myelination of corticobulbar pathways occurs earlier than the CST, between 24 and 28 weeks gestation. Unlike many CNS long tracts, myelination of the CST does not occur along all portions of the long axon simultaneously, but rather proceeds in a rostrocaudal direction (2-5). In the term neonate, the CST is relatively well myelinated in the corona radiata, internal capsule and in the middle third of the cerebral peduncle, but the pyramids at the base of the medulla oblongata appear unmyelinated at the light microscopic level and, when viewed in the electon microscope, individual axons have only one or two turns of myelin; in the spinal cord, these axons do not yet even show ultrastructural evidence of myelination. Both CST and CBT arise from widespread areas of contralateral neocortex, but the majority of fibers originate in motor regions of the frontal lobes.

Though the CST appears to be a single, large compact pathway, it is complex and mediates most of the impulses from the cerebellum via a synaptic way-station in the ventrolateral thalamic nucleus, and is the only means of expression of impulses from the corpus striatum and other basal telencephalic nuclei.

\section{Inhibitory effect on monosynaptic stretch reflexes}

The corticospinal tract is the second largest inhibitory pathway in the central nervous system; only the corpus callosum is larger. More than 80 percent of axons of the CST mediate an inhibitory effect on motor neurons of the spinal cord. Its net influence on the simple, monosynaptic myotatic or stretch reflex, a pure spinal cord segmental reflex that develops 25 weeks gestation or earlier in the human fetus (6), is to diminish its amplitude and dampen the oscillations that would otherwise result in clonus if the reflex were not inhibited. Overactive tendon stretch reflexes are a classical sign of corticospinal tract deficit in adults, and this feature of CST function in the newborn is no different. Normal neonates have relatively brisk reflexes, crossed adduction and sometimes one or two beats of ankle clonus, particularly when crying or agitated, that are all accepted as normal at this age. As the infant matures (i.e. his/her CST acquires myelin and its axons form more terminal ramifications), the stretch reflexes are more inhibited and become relatively less exaggerated that at birth, crossed adduction disappears and even unsustained neonatal clonus becomes a pathological sign.

Inhibition is one of the fundamental principles of how the nervous system works. Soon after winning the Nobel Prize in Medicine in 1963, Professor John Eccles, the Australian neurophysiologist who discovered inhibitory pathways in the CNS, was posed a question from someone in the audience at an international congress: Professor Eccles, how can the corticospinal tract be the most important central pathway mediating voluntary movement if it is almost entirely inhibitory? An analogy with which he responede provides keen in sight into how the brain works: The brain is not a painting; it is a sculpture. Michaelangelo did not create his magnificent Statue of David by starting with a massive block of crude stone and adding many more small pieces of stone. He indeed started with the block of stone, but by selectively chipping away small pieces, its coarseness was refined and the crude rock became a refined work of art. Excitation in the nervous system is the crude stone block; the CST selectively inhibits certain motor neurons to enable fine, refined movements and by reciprocally inhibiting muscles antagonistic to those being voluntarily contracted. Without such CST inhibition, we are left with spasticity and lack of precise movement or of reciprocal relaxation of antagonist muscles, i.e massive and diffuse excitation and loss of much voluntary motor control.

The spinal monosynaptic stretch reflex is already functional at birth, both by clinical examination and electrophysiologically using the H-reflex (7). Studies of human fetuses demonstrate that this reflex is already well developed at 25 weeks gestation, including the H-reflex in the triceps surae muscle, though at this age the latency is longer than in term neonates (6). It probably develops much earlier because the triceps surae monosynaptic stretch reflex first appears in the rat on embryonic day 19.5 (8), the same day that muscle spindles form (9). Muscle spindles form in human fetuses by 20 weeks gestation, and intrafusal myofibers are mature by 30 weeks (10). Primary afferent axons of descending bulbospinal and corticospinal pathways reach the ventral horns of the lumbar spinal cord on embryonic day 17.5 (11), so that this simple monosynaptic reflex is at least potentially modifiable by suprasegmental (i.e. upper motor neuron) control from the very beginning. In humans, some collaterals of sensory axons reach 


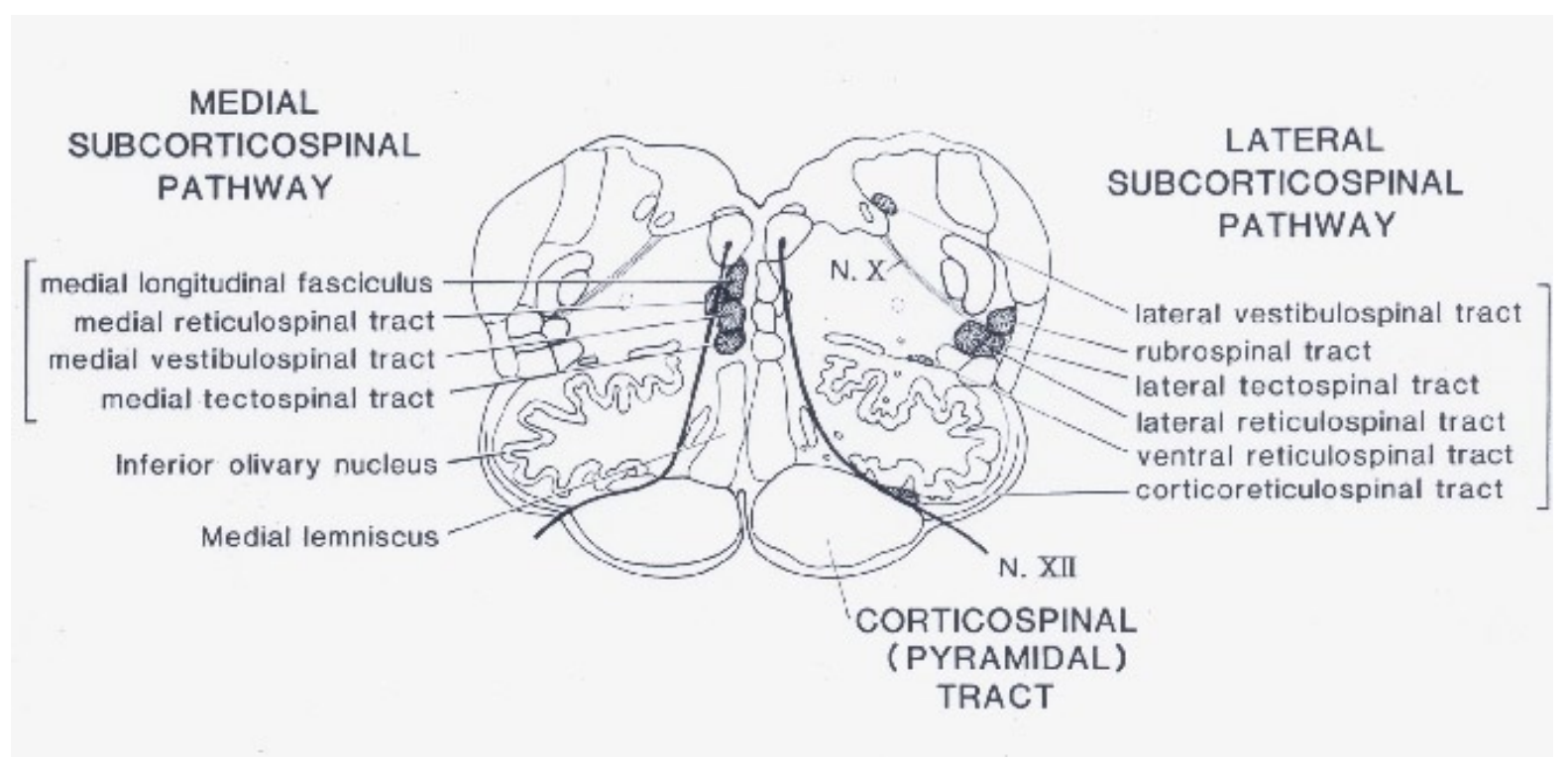

Figure 1. Schematic drawing of transverse section of medulla oblongata to indicate positions of bulbospinal and corticospinal tracts, and the grouping of Lawrence and Kuypers into "medial subcorticospinal" and "lateral subcorticospinal" pathways. The corticospinal tract is physiologically antagonistic to the medial subcortico-spinal pathway.

the ventral horns of the cervical spinal cord by 8 weeks gestation, and the lumbar region of the spinal cord slightly later, so that the reflex is potentially modifyable from the beginning in humans as well (12). It is, therefore, not surprising that the CST serves an inhibitory function in suppressing the amplitude and dampening oscillations (i.e. clonus) in the stretch reflexes of neonates.

\section{Influence on neonatal muscle tone and posture}

Posture and joint position are largely a function of resting muscle tone, unless there are physical restrictions of range of motion, such as adhesions or contractures of joints. The CST plays a key role in neonatal postures, in large part because its function as an antagonist to descending bulbospinal pathways. This physiological function was discovered by Lawrence and Kuypers, Canadian and British neurophysiologists. In the late 1960s and early 1970s, they studied effects of stimulation and selective destruction of specific descending upper motor neuron pathways in the brainstem of neonatal and adult monkeys $(13,14)$.

Lawrence and Kuypers grouped all descending bulbospinal pathways, as denoted above and shown in Figure 1, into two physiological pathways that they termed the "medial subcorticospinal" and "lateral subcorticospinal" (Figure 1), noting that both contained contributions from the same origins, such as the vestibular nuclei, reticular formation, inferior olivary and red nuclei and optic tectum (superior colliculus). Their findings can be summarized as follows: 1) the medial subcortico-spinal pathway mediates proximal extension and distal flexion, i.e. extension of the trunk, neck and limb girdles; 2) the lateral subcorticospinal pathway is inhibitory upon phasic alpha motor neurons mainly subserving paraspinal extensor muscles; 3) the corticospinal tract mediates proximal flexion and distal extension, in other words, antagonizes the effects of the medial subcorticospinal pathway.

These studies provide a physiological basis for understanding the postures we see clinically in infants with cortical impairment (hence CST dysfunction) with preserved brainstem function, regardless of the cause of the cortical impairment or whether it is due to potentially reversible metabolic depression without cellular death or irreversible due to neuronal necrosis. Opisthotonus, hypercontraction of extensor axial and cervical muscles and extension of the shoulders and hips, associated with fisting, strong flexion of the fingers and toes, and adduction of the thumbs across the palms (the "cortical thumb of Paine"), can now be readily interpreted as expression of the medial subcorticospinal pathway without antagonism of the CST to balance its effects (Figures 2 and 3). Flexion and adduction are neurologically the same, derived from the same pool of motor neurons; extension and abduction are similarly paired neurologically. The effects on axial muscles to produce opisthotonus denote more severe impairment that the effects on distal muscles, and most infants exhibit distal flexion without proximal extension.

The lateral subcorticospinal pathway of Lawrence and Kuypers probably preventsopisthotonus in some infants. A return of CST function in the neonate is 


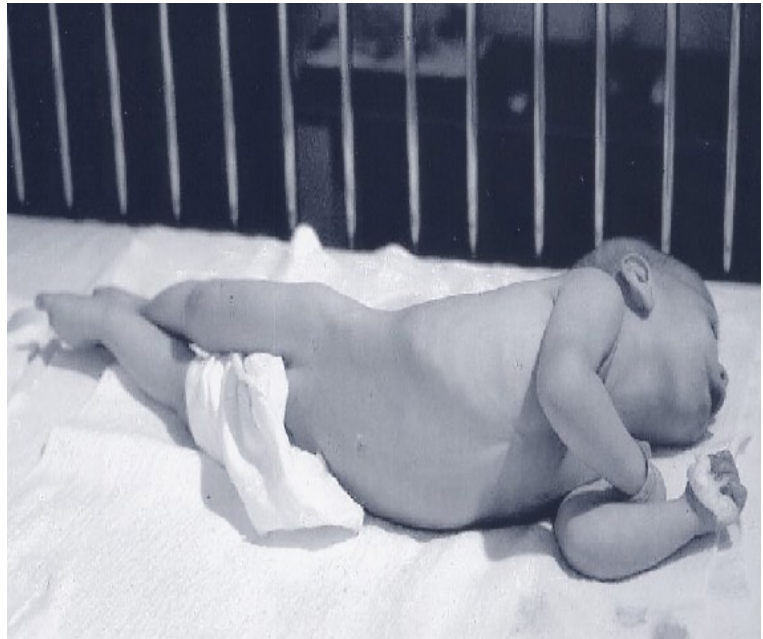

Figure 2. A 9-month-old infant, born at term, who suffered severe ischemic/hypoxic encephalopathy at birth. Note the opisthotonic posturing with hyper-extension of the spine and neck, extension of the hips, and tight flexion of the fingers. This classical posture is correlated with defective corticospinal antagonism of normal brainstem function (preserved medial subcorticospinal pathway) due to extensive laminar necrosis of the cerebral cortex and sparing of subcortical structures.

heralded by relaxation of the tightly fisted hands with extension of fingers and also by a return of tactile reflexes (see below).

From a neurological perspective, the important joints to observe are the proximal (trunk, neck and limb girdles) and distal (wrists, fingers, thumbs, toes). Intermediate joints, the elbows and knees, are less reliable indicators. Preterm infants often exhibit abduction of the hips into a frog-like posture (Figure 4), rather than extension with the legs together. Extension and abduction are neurologically the same, as noted above, and this difference probably is more related to the maturation of the hip joint itself and the posture of least resistance, than to any important neurological difference. Postures in the neonate also are influenced by vestibular input and stretch reflexes in cervical muscles (e.g. the asymmetrical tonic neck reflex) and by startle (e.g. the Moro reflex), but these are all mediated by the bulbospinal tracts.

Most neonates with acute impairment of CST function are diffusely hypotonic, including those with permanent cortical damage who evolve in a few months into spastic diplegia or classical "cerebral palsy". This hypotonia is centrally mediated and not due to peripheral neuropathy, myopathy or impaired neuromuscular junctions. It may be the expression of the cerebellum and subcortical telecephalic nuclei or inability of these structures to modulate muscle tone because of impaired CST function. Generalized muscular hypotonia also is one of the most constant neurological signs in infants with

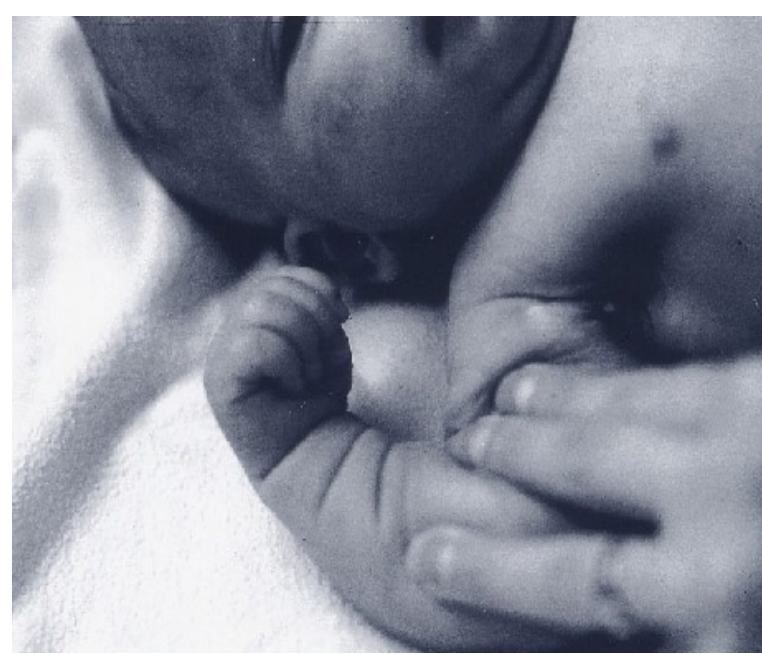

Figure 3. Hand of full-term neonate who suffered intrapartum asphyxia and had low Apgar scores, with subsequent clinical and EEG evidence of cerebral cortical impairment. There is strong flexion of wrist and fingers and adduction of the thumb across the palm. This is a typical distal posture indicating impaired CST function with preserved bulbospinal influences. This infant did not, however, have opisthotonus. He required gavage feedings and had neonatal seizures controlled with phenobarbital.

cerebellar hypoplasia, regardless of the etiological lesion (15), as well as in infants with damage to the basal ganglia due to bilirubin encephalopathy (kernicterus). The mechanism of cerebellar hypotonia, as shown in experimental studies in the monkey, is inhibition of fusimotor tone in muscle spindles (16). The cerebellum also influences histochemical differentiation of muscle between 20 and 28 weeks gestation, and children with cerebellar hypoplasia usually have a massive fiber type predominance or congenital muscle fiber-type disproportion $(17,18)$.

\section{Enhancement of tactile reflexes}

Tactile reflexes are reflexes in which cutaneous or mucus membrane stimulation is the afferent and muscle contraction is the efferent components of the reflex arc. Examples are the trunk incurvation reflex of Galant (stroking the infant's side causes the trunk of the body to curve toward the stimulus, a primitive reflex reliably present as early as 24 weeks gestation), the rooting response, the plantar response and the grasp. The most important tactile reflexes of the newborn are the suck and the swallow. All of these tactile reflexes are mediated at the brainstem or spinal cord level, but the corticospinal and corticobulbar tracts serve to reinforce or enhance them.

Any good neonatal nurse will tell you that infants who have impairment of the cerebral cortex (hence impairment of corticospinal tract 


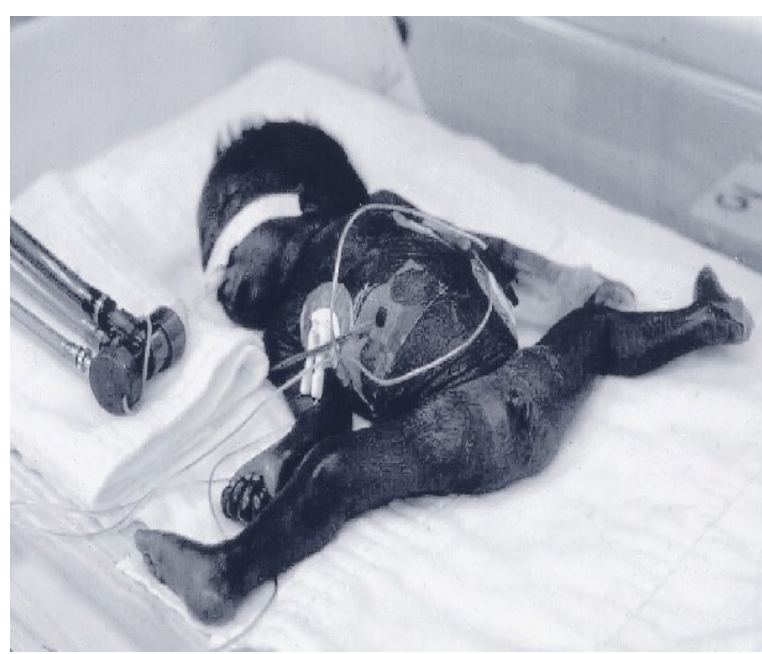

Figure 4. A 30-week premature infant who shows abduction, rather than extension, of the hips and extension of the shoulders. Abduction and extension are neurologically equivalent. Note also the fisting of the hands. The infant had fetal distress and required intubation at birth. He does not have germinal matrix or intraventricular hemorrhages.

function) have one universal feature in common: feeding difficulties. They suck poorly or not at all. Their coordination of swallowing is impaired so that they choke, aspirate, or fail to swallow altogether. It matters not whether the nature of the insult to the cerebrum is from is chemic/hypoxic encephalopathy, neonatal meningitis, subarachnoid hemorrhage, hydrocephalus, congenital infections or cerebral dysgenesis. Etiology is not important; acute or subacute cortical damage is the key factor. This failure of suck and swallow are due to the abrupt removal of the reinforcement function of the neonatal corticobulbar tract on these tactile reflexes. Careful neurological examination of the infant will reveal that other tactile reflexes also are absent or impaired: rooting, trunk incurvation, grasp and the extension of the fingers with their dorsal stroking (19).

Ironically, infants who have never had CST or CBT function in fetal life because the cerebral cortex has failed to develop or is only rudimentary, as in hydranencephaly, often do not show abnormal tactile reflexes and do suck and swollow. It is, therefore, the abrupt removal of this reinforcing influence when it was previously exerting an influence, even if only in fetal life, that determines the failure of tactile reflexes. Lack of reinforcement of the tactile swallow reflex is not the only cause of dysphagia in neonates who have suffered asphyxia in the perinatal period, however. There may be ischemia or microinfarcts in the tegmentum of the brainstem that impair the functions of important centers, including the nucleus ambiguus and nucleus and tractus solitarius.

\section{Mediator of individual finger movements}

One of the most important functions of the CST in the older child and adult is the mediation of fine individual finger movements. Children with spastic diplegia have great difficulty with refined, independent finger movements and loss of fine motor control. This CST function at maturity is related to its inhibitory function, as discussed above. However, even though newborns generally lack individual finger movements and have not yet acquired a pincer grasp, they do show some isolation of finger movements and not all spontaneous movements are done as if the hand were a mitten with all four fingers always moving together. Infants with cerebral cortical damage, by contrast, lose even these small isolated finger movements that normal term newborns show, again demonstrating that the CST is not a mute pathway at birth.

The red nucleus is another structure believed to be involved in individual finger movements. The red nucleus has two populations of neurons, small cells termed "parvocellular" part of the red nucleus, and large cells forming the "magnocellular" portion. The "parvocellular" part projects to the ipisilateral inferior olivary nucleus via the central tegmental tract, and forms one side of the "Guillain-Mollaret triangle" with the contralateral cerebellum. The magnocellular part contributes most fibers of the rubrospinal tract, but this tract only extends as far caudally as the cervical level of spinal cord in humans (20); the monkey is similar (21), though the rubrospinal tract extends to lumbar levels in the cat (22). This limited longitudinal extent in humans is sufficient to influence finger movements, controlled by muscles innervated mainly from ventral spinal roots C6-T1, and rubrospinal influences must be integrated with those of the CST. The corticorubral fibers that arise in sensory cortex are important in contact placing in kittens as well as in mature cats $(23,24)$, and may play a tactile role in individual finger movements in humans. A rubrobulbar projection from the magnocellular portion cross in the ventral tegmental decussation to synapse upon neurons of the facial nucleus, the principal sensory and spinal trigeminal nuclei, the small deep cerebellar nuclei (but not the dentate nucleus) and the medullary reticular formation $(22,25)$. The olivospinal tract is sparse in primates, though prominent in rodents. Some studies suggest that lesions of the spinal cord do not diminish the number of neurons in the red nucleus or of rubrospinal or corticospinal axons (26), but others demonstrate retrograde degeneration of the CST after human spinal cord injury (27).

\section{Relay of epileptic discharges originating in the cerebral cortex}

As in older children and adults, neonatal 
epileptiform activity originating in the cerebral cortex is mediated at brainstem and spinal cord levels via the CBT and CST, regardless of the etiology of the seizures. Whether subcortical foci of epileptiform activity can be transmitted to muscles through any of the bulbospinal tracts remains speculative and not well documented. Episodes of decerebrate posturing from function at the midbrain level are different from true epileptic activity and also from the opisthotonus of CST impairment, and should not be misinterpreted as "seizures".

\section{References}

1. Sarnat HB. Do the corticospinal and corticobulbar tracts mediate functions in the human newborn? Can J Neurol Sci 1989; 16: 157-160.

2. Yakovlev PI, Lecours AR. The myelination cycles of regional maturation of the brain. In: Minkowski A (ed). Regional Development of the Brain in Early Life. Philadelphia: FA Davis, 1967, pp 3-70.

3. Rorke LB, Riggs HE. Myelination of the Brain in the Newborn. Philadelphia: JB Lippincott, 1969.

4. Gilles FH. Myelination in the neonatal brain. Hum Pathol 1976; 7: 244-248.

5. Brody BA, Kinney HC, Kloman AS, Gilles FH. Sequence of central nervous system myelination in human infancy. I. An autopsy study of myelination. J Neuropathol Exp Neurol 1987; 46: 283-301.

6. Hakamada S, Hayakawa F, Kuno K, Tanaka R. Development of the monosynaptic reflex pathway in the human spinal cord. Brain Res 1988; 470: 239 246.

7. Vecchierini-Blineau MF, Guiheneuc P. Excitability of the monosynaptic reflex pathway in the child from birth to four years of age. J Neurol Neurosurg Psychiatry 1981; 44: 309-314.

8. Kudo N, Yamada T. Development of the monosynaptic stretch reflex in the rat: an in vitro study. J Physiol 1985; 369: 127-144.

9. Milburn A. The early development of muscle spindles in the rat. J Cell Sci 1973; 12: 175-195.

10. Sarnat HB. Ontogenesis of striated muscle. In: Polin RA, Fox WF, (eds). Fetal and Neonatal Physiology. Vol. 2. (2 ${ }^{\text {nd }}$ ed). Philadelphia: WB Saunders, 1998, pp 2226-2247.

11. Kudo N, Yamada T. Morphological and physiological studies of development of the monosynaptic reflex pathway in the rat lumbar spinal cord. J Physiol 1987; 389: 441-459.

12. Windle WF, Fitzgerald JE. Development of the spinal reflex mechanism in human embryos. J Comp Neurol 1937; 67: 493-509.

13. Lawrence DG, Kuypers HG. The functional organization of the motor system in the monkey. I. The effects of bilateral pyramidal lesions. Brain 1968; 91: 1-14.

14. Lawrence DG, Kuypers HG. The functional organization of the motor system in the monkey. II. The effects of lesions of the descending brain-stem pathways. Brain 1968; 91: 15-36.

15. Sarnat HB, Alcalá H. Human cerebellar hypoplasia. A syndrome of diverse causes. Arch Neurol 1980; 37: 300-305.

16. Gilman S. The mechanism of cerebellar hypotonia: An experimental study in the monkey. Brain 1969; 92: 621-638.

17. Sarnat HB. Le cerveau influence-t-il le développement musculaire du foetus humain? Can J Neurol Sci 1985; 12: 111-120.

18. Sarnat HB. Cerebral dysgeneses and their influence on fetal muscle development. Brain Dev 1986; 8: 495-499.

19. Modanlou HD. Extension reflex of fingers in the newborn. Pediatr Neurol 1988; 4: 66-67.

20. Nathan PW, Smith MC. The rubrospinal and central tegmental tracts in man. Brain 1982; 105: 233-269.

21. Kuypers HG, Lawrence DG. Cortical projections to the red nucleus and the brain stem in the Rhesus monkey. Brain Res 1967; 4: 151-188.

22. Courville J. Rubrobulbar fibers to the facial nucleus and the lateral reticular nucleus (nucleus of the lateral funiculus). An experimental study in the cat with silver impregnation methods. Brain Res 1966; 1: 317-337.

23. Amassian VE, Ross RJ. Developing role of sensorimotor cortex and pyramidal tract neurons in contact placing in kittens. J Physiol (Paris) 1978; 74: 165-184.

24. Batson DE, Amassian VE. A dynamic role of rubral neurons in contact placing by the adult cat. J Neurophysiol 1986; 56: 835-856.

25. Courville J, Brodal A. Rubro-cerebellar connections in the cat: an experimental study with silver impregnation methods. J Comp Neurol 1966; 126: 471-485.

26. Cheney PD, Hill-Karrer J, Belhaj-Saif A, McKiernan BJ, Park MC, Markario JK. Cortical motor areas and their properties: implications for neuroprosthetics. Prog Brain Res 2000; 128: 135-160.

27. Pasquale-Styles M, Marcillo A, Norenberg MD. Retrograde degeneration of the corticospinal tract in human spinal cord injury. J Neuropathol Exp Neurol 2003; 62: 568 (abstract). 